\title{
Sources of quantum waves
}

\author{
A. D. Baute ${ }^{1,2}$, I. L. Egusquiza ${ }^{1}$ and J. G. Muga ${ }^{2}$ \\ ${ }^{1}$ Fisika Teorikoaren Saila, Euskal Herriko Unibertsitatea, 644 P.K., 48080 Bilbao, Spain \\ ${ }^{2}$ Departamento de Química-Física, Universidad del País Vasco, Apdo. 644, Bilbao, Spain
}

\begin{abstract}
Due to the space and time dependence of the wave function in the time dependent Schrödinger equation, different boundary conditions are possible. The equation is usually solved as an "initial value problem", by fixing the value of the wave function in all space at a given instant. We compare this standard approach to "source boundary conditions" that fix the wave at all times in a given region, in particular at a point in one dimension. In contrast to the well-known physical interpretation of the initial-value-problem approach, the interpretation of the source approach has remained unclear, since it introduces negative energy components, even for "free motion", and a time-dependent norm. This work provides physical meaning to the source method by finding the link with equivalent initial value problems.
\end{abstract}

PACS: 03.65.-w

EHU-FT/0007

\section{INTRODUCTION}

The time dependent Schrödinger equation is commonly solved as an "initial value problem". In one dimension this means that the wave function $\psi(x, t)$ is fixed for all $x$ at a reference time, usually $t=0$. However, it is also possible to use different boundary conditions by fixing $\psi(x, t)$ at a space point, say $x=0$, at all times. This "source" approach has been followed to study the arrival time in quantum mechanics [1], or characteristic propagation velocities and times ("tunneling times") in evanescent conditions [2 8]. In all these applications the wave function is assumed to vanish for $x \geq 0$ and $t<0$,

$$
\psi_{s}(x, t)=0, \quad x \geq 0, \quad t<0,
$$

the potential is zero or constant for $x \geq 0$ and $t>0$, and the solution of the Schrödinger equation is sought for the positive half-line and positive times, $\psi_{s}(x \geq 0, t>0)$. We shall also limit the present discussion to these premises, and use the subscript $s$ to denote the solutions that obey Eq. (11), computed for the space-time domain $D_{+} \equiv\{x \geq 0 ; t>0\}$. They have been invariably written, up to constant factors and notational changes, as

$$
\psi_{s}(x, t)=\frac{1}{h^{1 / 2}} \int_{-\infty}^{\infty} d E e^{i x p_{+} / \hbar-i E t / \hbar} \chi_{s}(E)
$$

where

$$
\chi_{s}(E)=\frac{1}{h^{1 / 2}} \int_{-\infty}^{\infty} d t \psi_{s}(x=0, t) e^{i E t / \hbar}
$$

is the energy Fourier transform of the given "source signal" $\psi_{s}(x=0, t)$ and $p_{+}=(2 m E)^{1 / 2}$, with the branch cut taken along the negative imaginary axis of $E$. Because of Eq. (il), $\chi_{s}(E)$ is an analytical function of $E$ for $\operatorname{Im} E>0$; however, this does not guarantee the absence of singularities of $\chi_{s}(E)$ for real $E$, additional to the branch point. In fact, the integral over $E$ is to be understood as going above the real axis; alternatively, $E \rightarrow E+i 0$ is to be substituted in the expressions above.

It is easy to check by substitution that Eq. (2) satisfies the Schrödinger equation. Actually Allcock derived Eq. (2) as the most general form compatible with Eq. (11) [1]. However he did not analyze its connection with initial value problems, or the meaning of the negative energies, so its physical interpretation has remained unclear. What are the negative energy components? How should such a wave be normalized? In time instead of position? Indeed, should it be normalized? Is the source to be interpreted as a multi-particle source? Except for the detailed analysis by Allcock, most authors have used the source boundary conditions without discussion, or as a simplifying approach whose actual physical content was questionable, as reflected, for example, in Moretti's words [4]: "The model is surely more suited in the electromagnetic case; for a quantum particle, it seems more satisfactory a localization in a given region, at a given time, rather than a "creation" during a given time interval, at a fixed position. [....] (The model) is chosen by reasons of simpler mathematics".

A "source signal" such as [2 [8] 


$$
\psi_{s}(x=0, t)=e^{-i \omega_{0} t} \Theta(t)
$$

leads to an infinite norm as $t \rightarrow \infty$ for $\omega_{0}>0$. An interpretation of such an infinite norm might only be possible by invoking a multi-particle approach which we shall not pursue here. Instead, if we keep within the one particle picture, it becomes clear that not all "signals" $\psi_{s}(x=0, t)$ may be allowed physically. This does not mean that the results obtained with Eq. (4) are meaningless. This signal may be an idealized approximation during a certain time interval of an actual signal, and it is also an elementary component of an arbitrary physical signal. Our basic philosophy here is that the physical interpretation of Eq. (2) must rest on a process where a wave packet located in the left half-line is liberated at $t=0$ and crosses, in general only partially, to the right half-line. This was the point of view of Allcock too [1], although our scope will differ from his work. There must be a link between a physically valid $\psi_{s}$ and some initial value problem, and the objective of this paper is to find and discuss such a link and the meaning of the negative energies in Eq. (2). The consequences on the possibility to define time-of-arrival distributions in quantum mechanics are also examined.

\section{EQUIVALENCE BETWEEN INITIAL-VALUE AND SOURCE BOUNDARY CONDITIONS}

\section{A. Free motion on the full line}

We shall solve in this section the initial value problem corresponding to a wave restricted fully to the left half-line up to $t=0$, in the interval $[a, b]$, with $a<b<0$, by an appropriate time-dependent potential that vanishes for $t \geq 0$. The resulting wave $\psi_{i v}$ is only free from $t=0$ onwards. It is also useful to introduce a related wave $\psi_{f}$ which evolves freely at all times, positive and negative, and is equal to $\psi_{i v}$ for $t \geq 0$,

$$
\psi_{f}(x, t)=\int_{a}^{b} d x^{\prime}\left\langle x\left|U\left(t, t^{\prime}=0\right)\right| x^{\prime}\right\rangle \psi_{i v}\left(x^{\prime}, t^{\prime}=0\right), \quad \forall t .
$$

Here $U\left(t, t^{\prime}\right)=\exp \left[-i H_{0}\left(t-t^{\prime}\right) / \hbar\right]$ is the unitary evolution operator for the free-motion Hamiltonian $H_{0}$,

$$
\left\langle x\left|U\left(t, t^{\prime}\right)\right| x^{\prime}\right\rangle=\left[\frac{m}{i h\left(t-t^{\prime}\right)}\right]^{1 / 2} e^{i m\left(x-x^{\prime}\right)^{2} / 2 \hbar\left(t-t^{\prime}\right)} .
$$

By setting the following source signal,

$$
\psi_{s}(x=0, t)=\psi_{i v}(x=0, t) \Theta(t)=\psi_{f}(x=0, t) \Theta(t),
$$

the corresponding $\psi_{s}(x, t)$ is obtained via Eq. (2). We shall now establish the equality between $\psi_{s}$ and $\psi_{f}$ for positive times and positions when the "boundary condition" of Eq.(17) is imposed on $\psi_{s} . \psi_{f}(x, t)$ may also be written as a momentum integral,

$$
\psi_{f}(x, t)=\frac{1}{h^{1 / 2}} \int_{-\infty}^{\infty} d p e^{i x p / \hbar} e^{-i E t / \hbar} \tilde{\psi}_{f}(p),
$$

where $E=p^{2} / 2 m$, and

$$
\tilde{\psi}_{f}(p)=\frac{1}{h^{1 / 2}} \int_{a}^{b} d x e^{-i p x / \hbar} \psi_{f}(x, t=0) .
$$

It can be separated into positive and negative momentum components, $\psi_{f}(x, t)=\psi_{f,+}(x, t)+\psi_{f,-}(x, t)$, where

$$
\psi_{f, \pm}(x, t)=\frac{1}{h^{1 / 2}} \int_{-\infty}^{\infty} d p e^{i x p / \hbar} e^{-i E t / \hbar} \tilde{\psi}_{f}(p) \Theta( \pm p) .
$$

The positive momentum integral is now rewritten in terms of the energy variable $E=p^{2} / 2 m$,

$$
\psi_{f,+}(x, t)=\frac{1}{h^{1 / 2}} \int_{0}^{\infty} d E e^{i p x / \hbar} e^{-i E t / \hbar}\left(\frac{m}{2 E}\right)^{1 / 2} \tilde{\psi}_{f}(\sqrt{2 m E}) .
$$

To put $\psi_{f,-}$ in a similar form, note that, because of the restriction of the initial state within the left half-line at $t=0$, its momentum representation $\tilde{\psi}_{f}(p)$, Eq. (9), is analytical in the complex plane $p$, and decays to zero as $|p| \rightarrow \infty$ in 
the upper half-plane. These two properties and the decaying behavior of the exponentials of Eq. (8) in the second quadrant allow to substitute the integral along the negative real axis by an integral along the positive imaginary axis (there are no poles and the integral along a large arc at infinity is zero),

$$
\psi_{f,-}(x, t)=\frac{i}{h^{1 / 2}} \int_{\infty}^{0} d \gamma e^{-x \gamma / \hbar} e^{i \gamma^{2} t / 2 m \hbar} \tilde{\psi}_{f}(i \gamma) \quad x \geq 0, t>0
$$

where we have used $p=i \gamma(\gamma \geq 0)$, and $\tilde{\psi}_{f}(i \gamma)$ is defined through Eq. (9). Changing now to the energy variable $E=-\gamma^{2} /(2 m)$

$$
\psi_{f,-}(x, t)=-\frac{i}{h^{1 / 2}} \int_{-\infty}^{0} d E e^{i x p_{+} / \hbar} e^{-i E t / \hbar}\left(\frac{m}{-2 E}\right)^{1 / 2} \tilde{\psi}_{f}\left[i(-2 m E)^{1 / 2}\right], \quad x \geq 0, t>0 .
$$

Adding Eq. (11) and Eq. (13) we can now write $\psi_{f}(x, t)$ for $x \geq 0, t>0$ in the form of the right hand side of Eq. (2),

$$
\psi_{f}(x, t)=\frac{1}{h^{1 / 2}} \int_{-\infty}^{\infty} d E e^{i x p_{+} / \hbar} e^{-i E t / \hbar} \chi_{f}(E), \quad x \geq 0, t>0,
$$

where

$$
\chi_{f}(E)=(m / 2 E)^{1 / 2} \tilde{\psi}_{f}\left[(2 m E)^{1 / 2}\right]
$$

As before, the cut defining the square root branch is taken at the negative imaginary axis of $E$, so that the square roots are positive imaginary numbers for negative energies. It is also useful to write Eq. (14) as an integral in the momentum plane,

$$
\psi_{f}(x, t)=\frac{1}{h^{1 / 2}} \int_{L} d p e^{i p x / \hbar} e^{-i E t / \hbar} \tilde{\psi}_{f}(p), \quad x \geq 0, t>0,
$$

where the contour is an $L$-shaped line that goes downwards along the positive imaginary axis and rightwards along the positive real axis. Let us now define the function $\phi$ by the integral in Eq. (16) without any restriction on $x$ or $t$,

$$
\phi(x, t) \equiv \frac{1}{h^{1 / 2}} \int_{L} d p e^{i p x / \hbar} e^{-i E t / \hbar} \tilde{\psi}_{f}(p),
$$

and analyze its behavior for $x \geq 0$ and $t<0$. The contour $L$ can be closed by a large arc in the first quadrant which does not contribute to the integral. By Cauchy's theorem, $\phi(x, t)$ is zero for $x \geq 0$ and $t<0$. In particular, this means that $\phi(x=0, t)=\psi_{s}(x=0, t)$.

We may also write Eq. (17) as an integral along the real energy axis,

$$
\phi(x, t)=\frac{1}{h^{1 / 2}} \int_{-\infty}^{\infty} d E e^{i x p_{+} / \hbar} e^{-i E t / \hbar} \chi_{f}(E)
$$

and solve for $\chi_{f}(E)$ by Fourier transform,

$$
\chi_{f}(E)=\frac{1}{h^{1 / 2}} \int_{-\infty}^{\infty} d t e^{i E t / \hbar} \phi(x=0, t) .
$$

However $\phi(x=0, t)=\psi_{s}(x=0, t)$, so that $\chi_{f}(E)=\chi_{s}(E)$, and therefore $\psi_{s}(x, t)=\psi_{f}(x, t)$ in the space-time domain $D_{+}$when the boundary condition specified in Eq. (7) is satisfied at $x=0$, and the state is initially localized as indicated above.

Since in many instances the given function is the signal $\psi_{s}(x=0, t)$ rather than its Fourier transform, it is also convenient to express Eq. (2) as an integral over time in terms of the signal. By inserting Eq. (3) and changing the order of integration, we find the appealing expression

$$
\psi_{s}(x, t)=\int_{0}^{t} d t^{\prime} K_{+}\left(t, x ; t^{\prime}, x^{\prime}=0\right) \psi_{s}\left(x^{\prime}=0, t^{\prime}\right)
$$

where the kernel is given by 


$$
K_{+}\left(t, x ; t^{\prime}, 0\right)=\frac{1}{h} \int_{-\infty}^{\infty} d E e^{i p_{+} x / \hbar} e^{i E\left(t^{\prime}-t\right) / \hbar}=\frac{1}{h m} \int_{L} d p p e^{-i p^{2}\left(t-t^{\prime}\right) /(2 m \hbar)} e^{i p x / \hbar} .
$$

Notice that $K_{+}\left(t, 0 ; t^{\prime}, 0\right)=\delta\left(t^{\prime}-t\right)$. Let us evaluate Eq. (21) for $x \geq 0$ along the $L$-shaped contour in the momentum plane. For $\left(t-t^{\prime}\right)<0$ the contour may be closed by a large arc at $\infty$ in the first quadrant, which does not contribute to the integral. Since no pole is enclosed, the integral is zero. This explains the upper limit $t$ in Eq. (20).

For $\left(t-t^{\prime}\right)>0$ the part of the contour in the imaginary axis may be deformed into the negative real axis. This allows the identification of $K_{+}$with the derivative of the ordinary propagator,

$$
\begin{aligned}
K_{+}\left(t, x ; t^{\prime}, 0\right) & =\int_{-\infty}^{\infty} d p \frac{p}{h m} e^{i p^{2}\left(t^{\prime}-t\right) /(2 m \hbar)} e^{i p x / \hbar} \\
& =\frac{\hbar}{m i} \frac{d}{d x}\left\langle x\left|U\left(t, t^{\prime}\right)\right| 0\right\rangle \\
& =\left[\frac{m}{i h\left(t-t^{\prime}\right)^{3}}\right]^{1 / 2} x e^{\frac{i m x^{2}}{2 \hbar\left(t-t^{\prime}\right)}} \quad\left(t>t^{\prime}, x \geq 0\right) .
\end{aligned}
$$

A related result was obtained by Allcock on his way to Eq. (2) [1], by considering the retarded free propagator. The expression of Allcock associates the source wave function to a time integral of the wave function at the source point times the free propagator, with a double-sided spatial derivative in between. It can be shown that it is equivalent to Eq. (20). An analogous relation is also obtained in three dimensions, with similar techniques but rather different objectives, by Mangin-Brinet et al [9], relating the wave function in one region of spacetime with the convolution over that region of the initial wave function with the propagator, plus a surface term with a double-sided gradient.

The relation found between $\psi_{s}$ and $\psi_{f}$, and Eq. (15) are the main results of this section. They provide a physical interpretation of the source wave function in terms of an initial value problem when there is free motion after the initial state is released at $t=0$. Note that the knowledge of the signal $\psi_{s}(x=0, t)$, or of its Fourier transform $\chi_{s}(E)$, allows to recover the negative momentum part of the initial state $\psi_{i v}(t=0)$ for the corresponding initial value problem only insofar as the amplitude $\tilde{\psi}_{f}(p)$, known in principle along the positive real and imaginary axis, can be analytically continued into the negative real axis.

In this way, the normalizability required of $\psi_{f}$ (or $\psi_{i v}$, for that matter), is translated into a property of $\psi_{s}$, which thus acquires the usual meaning of a wave-function in quantum mechanics, that is, as a probability amplitude. It should be observed that not all functions of the form of Eq. (2) can have this meaning: only those that are indeed related to a given $\psi_{i v}$ or $\psi_{f}$ can be thus understood.

We may now see the origin of the negative energies in $\psi_{s}$ : the contribution of the evanescent waves in the source solution, Eq. (2), is exactly equal to the contribution of negative momenta in the corresponding freely moving wave packet $\psi_{f}$, Eq. (8). The change of variable and the shift in the path of integration lead to what could be termed apparent evanescent waves, which in fact can be understood as an artifact of the Fourier-Laplace transformation in time. It may be surprising from a classical perspective that such a negative momentum contribution exists at positive times and positions, considering that the wave packet is entirely localized on the left at $t=0$. In quantum mechanics, however, the negative momentum (equivalently, evanescent or negative energy) contribution is always present, since $\tilde{\psi}_{f,-}(p)$ is restricted to a half line in momentum space and therefore $\psi_{f,-}(x)$ is necessarily different from zero from $-\infty$ to $+\infty$ except possibly at a finite number of points. It is true however that the influence of $\psi_{f,-}$ at $x>0$ diminishes with the distance to the origin. This is illustrated in Figures 1 and 2, that show the probability densities $\left|\psi_{f}(x, t)\right|^{2}$ and $\left|\psi_{f, \pm}(x, t)\right|^{2}$ versus $x$ at two different instants. The state is initially (at $t=0$ ) the ground state of an infinite well between $a$ and $b, a<b<0$, and has zero average momentum, $\langle p\rangle=0$. Its time evolution may be expressed in terms of know functions, see the Appendix. Note the long tails of $\left|\psi_{f, \pm}(x, t=0)\right|^{2}$ beyond $[a, b]$ in Figure 1 , and the important interference pattern between $\psi_{f,+}$ and $\psi_{f,-}$ in Figure 2. 


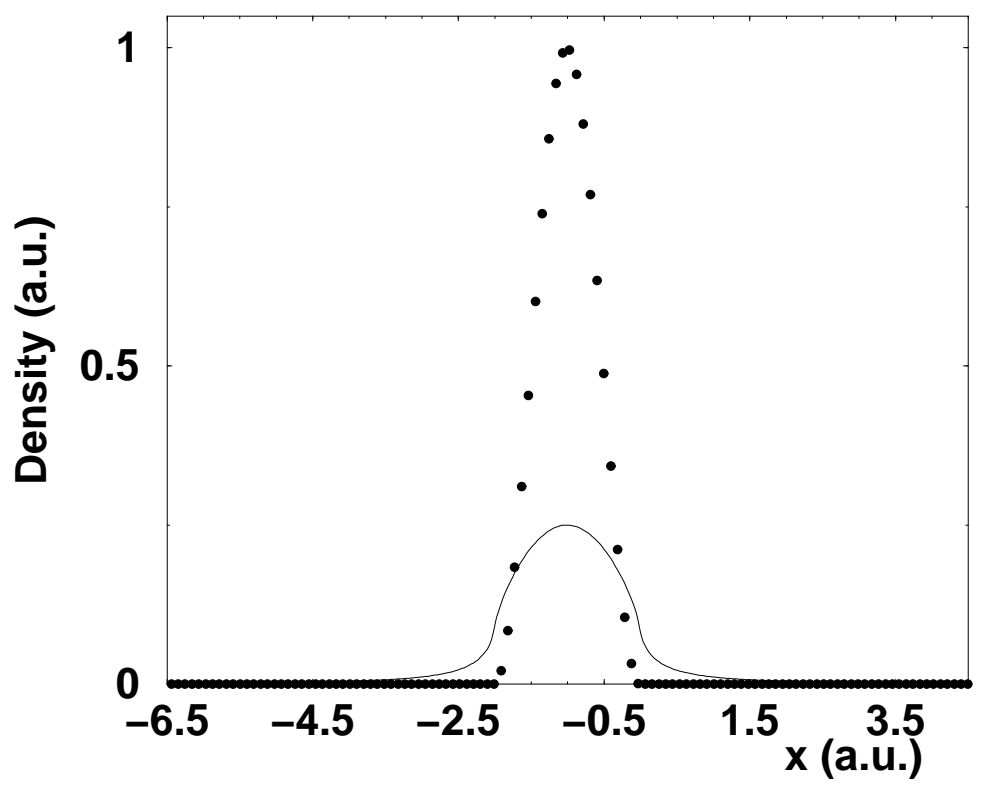

FIG. 1. $\left|\psi_{f}(x, t)\right|^{2}$ (dotted line), $\left|\psi_{f,+}(x, t)\right|^{2}$ (solid line), and $\left|\psi_{f,-}(x, t)\right|^{2}$ (dashed line, indistinguishable from the solid line) versus $x$ for $t=0$. The initial state is the ground state of an infinite well between $a=-2.01$ and $b=-0.01$, see Eq. A1). Here and in all figures, atomic units (a.u.) are used, $m=1, \hbar=1$.

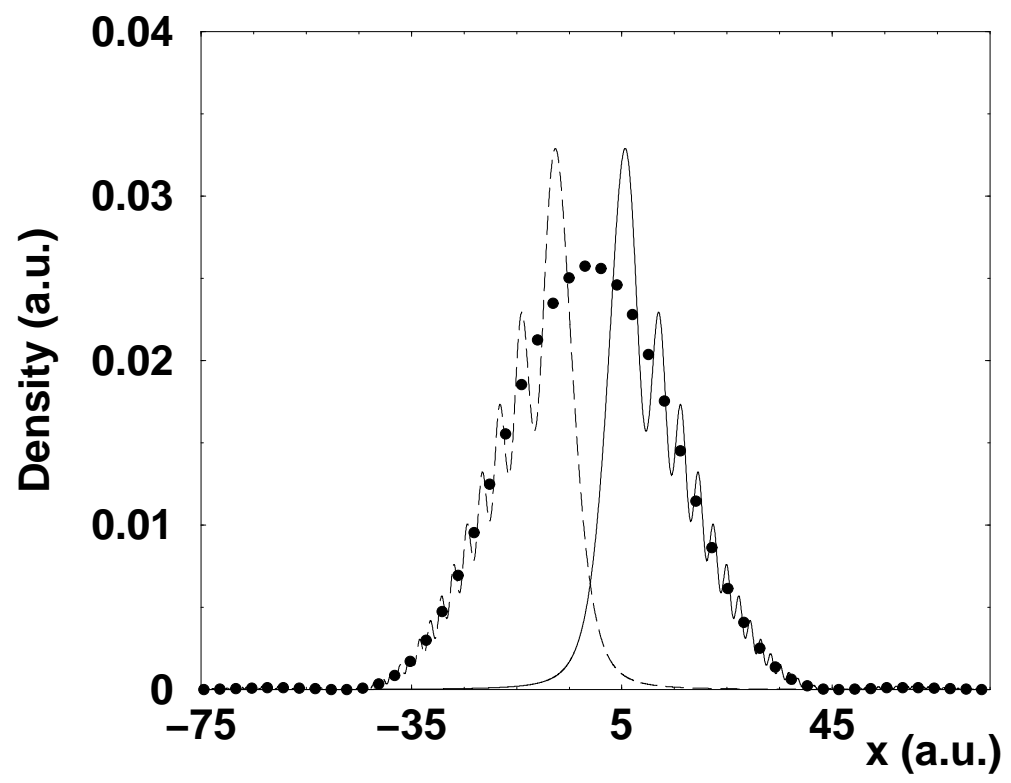

FIG. 2. Same as Fig. 1, but for $t=10$.

\section{B. Generalization for potentials that vanish outside an interval}

The above results can be generalized for an arbitrary cut-off potential profile that vanishes outside an interval $[c, d]$, located between the initial state and the source point $x=0$, i.e. such that $a<b \leq c \leq d \leq 0$. The time evolution of the wave, $\psi_{b}$, for $x \geq d$ is given by 10,11

$$
\psi_{b}(x, t)=\frac{1}{h^{1 / 2}} \int_{\Omega} d p e^{i p x / \hbar} e^{-i E t / \hbar} T(p) \tilde{\psi}_{b}(p) \quad x \geq d,
$$

(to be compared with Eq. (8)), where $T(p)$ is, for $p>0$, the transmission amplitude, and its analytical continuation elsewhere; similarly to Eq. (9), $\tilde{\psi}_{b}(p)=h^{-1 / 2} \int_{a}^{b} d x e^{-i p x / \hbar} \psi_{b}(x, t=0)$. The contour $\Omega$ goes from $-\infty$ to $\infty$. If there 
are bound states it passes above the corresponding poles of $T(p)$ in the positive imaginary axis. $T(p)$ is analytical in the upper half plane except in these points. Moreover it tends to one as $|p| \rightarrow \infty[12$. This means that the same manipulations done for the free motion wave function to write it in the form of $\psi_{s}$ can now be performed, but instead of $\tilde{\psi}\left[(2 m E)^{1 / 2}\right]$ in Eq. (15) one must write $T\left[(2 m E)^{1 / 2}\right] \tilde{\psi}\left[(2 m E)^{1 / 2}\right]$ in the more general case. The same arguments that have enabled us to identify $\psi_{f}$ and $\psi_{s}$ in $D_{+}$when the boundary condition of Eq. (7) is satisfied are now applicable to identify $\psi_{s}(x, t)$ and $\psi_{b}(x, t)$ in the same domain when

$$
\psi_{s}(x=0, t)=\psi_{b}(x=0, t) \Theta(t)
$$

is imposed as the signal at the source point $x=0$.

In any case free motion or motion with a localized potential barrier in the full line do not easily allow to construct source signals dominated by negative energy components. But these are precisely the signals of interest to study propagation velocities and characteristic times for evanescent conditions [2 8]. The way to construct them and their relation to initial value problems are examined in the next subsection.

\section{C. "Step potentials" with different asymptotic levels}

Here we shall show the connection between source wave functions and initial value problems for the simple step potential,

$$
V(x)=\Theta(x) V_{0}, \quad V_{0}>0
$$

as well as any other cut-off "step" potentials between $c$ and $d$, such that $V(x>d)=V_{0}$ and $V(x<c)=0$. As before, we assume that the initial state is localized between $a$ and $b$, with $a<b \leq c \leq d \leq 0$. Following the method used in [10] to write Eq. (23), it is possible to write the wave function for $x>0$, after lengthy manipulations [11], as

$$
\psi_{\text {step }}(x, t)=\frac{1}{h^{1 / 2}} \int_{\Omega} d p e^{-i E t / \hbar} e^{i q x / \hbar} T^{l}(p) \tilde{\psi}_{s t e p}(p),
$$

where $\tilde{\psi}_{\text {step }}(p)=h^{-1 / 2} \int_{a}^{b} d x e^{-i p x / \hbar} \psi_{\text {step }}(x, t=0)$. This is an exact expression, where $p$ is the momentum with respect to the lower level of the step; $E=p^{2} / 2 m$, and $q$ is the momentum with respect to the upper level. For real $p$, it is given by

$$
q= \begin{cases}-\left(p^{2}-p_{0}^{2}\right)^{1 / 2} & p<-p_{0} \\ i\left(p_{0}^{2}-p^{2}\right)^{1 / 2} & -p_{0}<p<p_{0} \\ \left(p^{2}-p_{0}^{2}\right)^{1 / 2} & p>p_{0}\end{cases}
$$

Here and in the following $p_{0}$ equals $\left(2 m V_{0}\right)^{1 / 2}$. For arbitrary values of $p$ in the complex plane, $q=\left(p^{2}-p_{0}^{2}\right)^{1 / 2}$ with the understanding that the two branch points at $p= \pm p_{0}$ are joined by the shortest branch cut, slightly displaced below the real axis. In particular, for a point $p=i \gamma(\gamma>0)$ the corresponding $q$ is $i\left(\gamma^{2}+p_{0}^{2}\right)^{1 / 2}$. $T^{l}(p)$ is, for $p>p_{0}$, the transmission amplitude for left incidence, and its analytical continuation elsewhere. As in the previous subsection, the contour $\Omega$ goes from $-\infty$ to $\infty$ passing above the branch cut and possible bound-state poles of $T^{l}(p)$ on the imaginary axis.

The integral in Eq. (26) may also be written in the $q$-plane as

$$
\psi_{\text {step }}(x, t)=\frac{1}{h^{1 / 2}} \int_{C} d q e^{i q x / \hbar} e^{-i E t / \hbar} \frac{q}{p} T^{l}(p) \tilde{\psi}_{s t e p}(p),
$$

where now $p=\left(q^{2}+p_{0}^{2}\right)^{1 / 2}$ has two branch points at $q= \pm i p_{0}$ joined by a branch cut. The contour $C$ goes from $-\infty$ to $+\infty$ passing above the part of branch cut in the positive imaginary axis and above the poles of $T^{l}(p)($ Note that whenever there are bound-state poles they are above the branch point at $\left.q=i p_{0}\right)$. It is possible, as in the previous section, to deform the contour in the second quadrant so that the final contour has an $L$ shape, going from $i \infty$ to zero passing to the right of the poles, to the right of the branch cut $\left(p>0\right.$ in the interval from $q=i p_{0}$ to $q=0$ ), and from zero to $\infty$ along the positive real axis, see Figure 3,

$$
\psi_{\text {step }}(x, t)=\frac{1}{h^{1 / 2}} \int_{L} d q e^{i q x / \hbar} e^{-i E t / \hbar} \frac{q}{p} T^{l}(p) \tilde{\psi}_{\text {step }}(p) .
$$




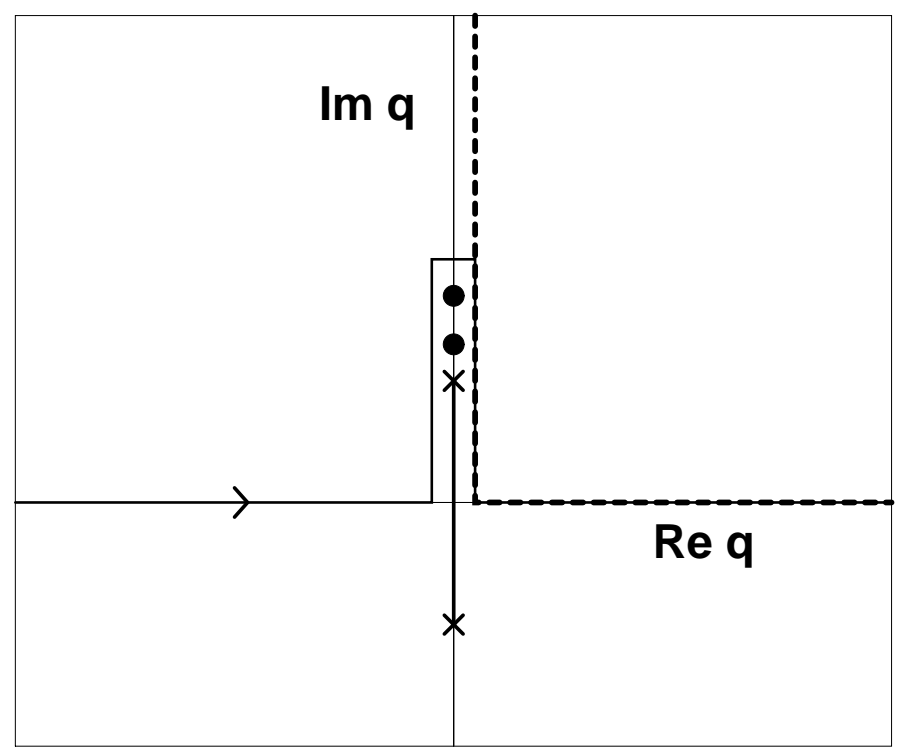

FIG. 3. Integration contours $C$ (solid line) and $L$ (dashed line) in the $q$-plane. The crosses represent branch points joined by a branch cut. The circles on the imaginary axis are bound states.

Finally, we set the zero of energy at the upper level of the potential profile using the change of variable $E^{\prime}=$ $E-V_{0}=q^{2} / 2 m$, and define the wave that satisfies the Schrödinger equation for this new level in terms of the wave function written for the lower level, $\psi_{\text {step }}^{\prime}(x, t)=e^{i V_{0} t / \hbar} \psi_{\text {step }}(x, t)$,

$$
\begin{aligned}
\psi_{\text {step }}^{\prime}(x, t) & =\frac{1}{h^{1 / 2}} \int_{L} d q e^{i q x / \hbar} e^{-i E^{\prime} t / \hbar} \frac{q}{p} T^{l}(p) \tilde{\psi}_{\text {step }}^{\prime}(p) \\
& =\frac{1}{h^{1 / 2}} \int_{-\infty}^{\infty} d E^{\prime} e^{i q x / \hbar} e^{-i E^{\prime} t / \hbar} \frac{m}{p} T^{l}(p) \tilde{\psi}_{\text {step }}^{\prime}(p) .
\end{aligned}
$$

We have thus expressed $\psi_{\text {step }}^{\prime}$ in the form of the source wave function $\psi_{s}$. The same arguments used to find the equality of $\psi_{f}$ and $\psi_{s}$ in $D_{+}$may now be applied to identify $\psi_{s}$ and $\psi_{s t e p}$. In particular,

$$
\chi_{s}\left(E^{\prime}\right)=\frac{m}{p} T^{l}(p) \tilde{\psi}_{s t e p}^{\prime}(p)
$$

where $p=\left[2 m\left(E^{\prime}+V_{0}\right)\right]^{1 / 2}$, when

$$
\psi_{s}(x=0, t)=\psi_{\text {step }}(x=0, t) \Theta(t) .
$$

We have performed a numerical test with the simplest step potential, Eq. (25). In this case,

$$
T^{l}(p)=\frac{2 p}{p+q} .
$$

The state is initially localized in the lower level of the step and sent towards the step. It is the same state used for Figures 1 and 2, but boosted with a non zero momentum below the step threshold. Figures 4 and 5 show the real and imaginary parts of $\chi_{s}\left(E^{\prime}\right)$ calculated by Fast Fourier Transform of the signal $\psi_{\text {step }}^{\prime}(x=0, t) \Theta(t)$, which has been obtained numerically by solving the time dependent Schrödinger equation with the Split Operator Method. Note the clear dominance of negative energy components. The curves shown are indistinguishable from the real and imaginary parts of the the right hand side of Eq. (31). 


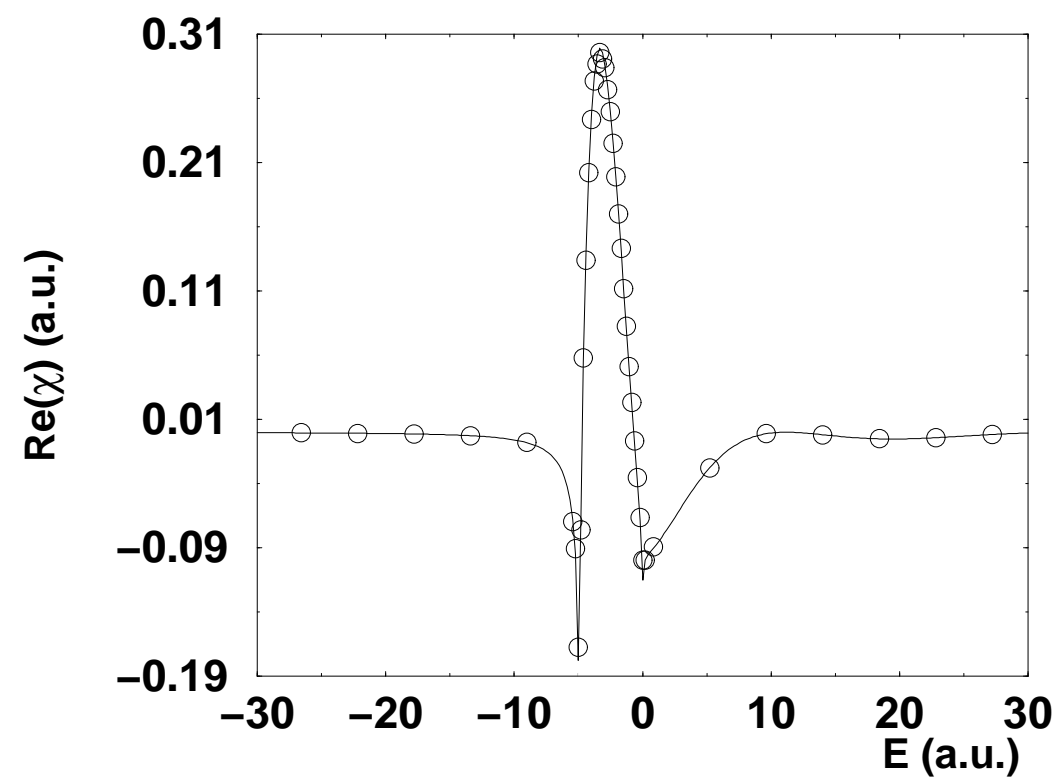

FIG. 4. Real parts of $\chi_{s}\left(E^{\prime}\right)$ (circles) and $\frac{m}{p} T^{l}(p) \tilde{\psi}_{\text {step }}^{\prime}(p)$ (solid line) for the step potential $\left(V_{0}=5\right)$. The initial state is the same one used in Figures 1 and 2, but shifted in momentum so that $\langle p\rangle=1$.

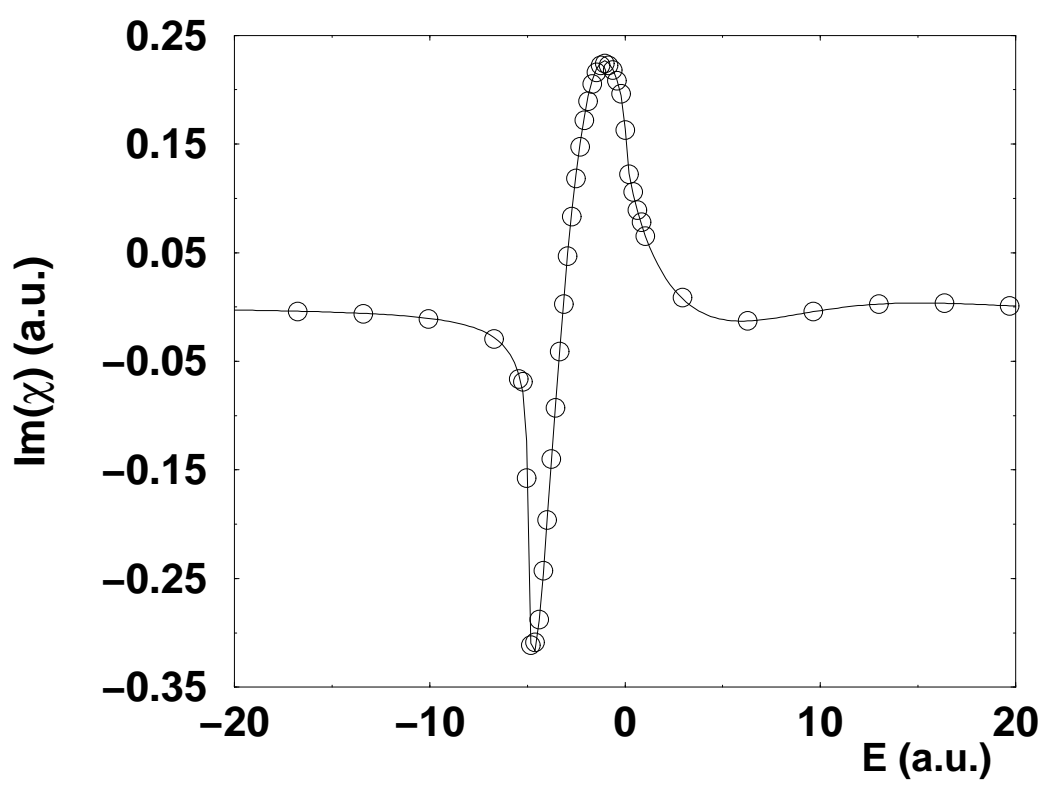

FIG. 5. Imaginary parts of $\chi_{s}\left(E^{\prime}\right)$ (circles) and $\frac{m}{p} T^{l}(p) \tilde{\psi}_{\text {step }}^{\prime}(p)$ (solid line) for the step potential $\left(V_{0}=5\right)$. Initial state as in Figure 4 .

All the connections made in this section between the momentum amplitude $\tilde{\psi}_{f}(p)$ and the energy Fourier transform of the source signal at $x=0$ have required a wave packet strictly localized between $a$ and $b$, in order to perform contour deformations. It is intuitively clear, however that relaxing this condition cannot lead to a completely different behavior in the relevant energy region. To test this claim, we have also calculated the real and imaginary parts of $\chi_{s}\left(E^{\prime}\right)$ by Fast Fourier Transform of $\psi_{\text {step }}^{\prime}(x=0, t) \Theta(t)$, which is obtained by solving the time dependent Schrödinger equation for an initial Gaussian state with negligible overlap with the positive- $x$ region; this is compared with the right hand side of Eq. (31) in Figures 6 and 7. Clearly, the right hand side of Eq. (31), obtained directly from the initial state, reproduces the Fourier transform in the important energy region, but for very negative values of $E^{\prime}$ they do not coincide. 


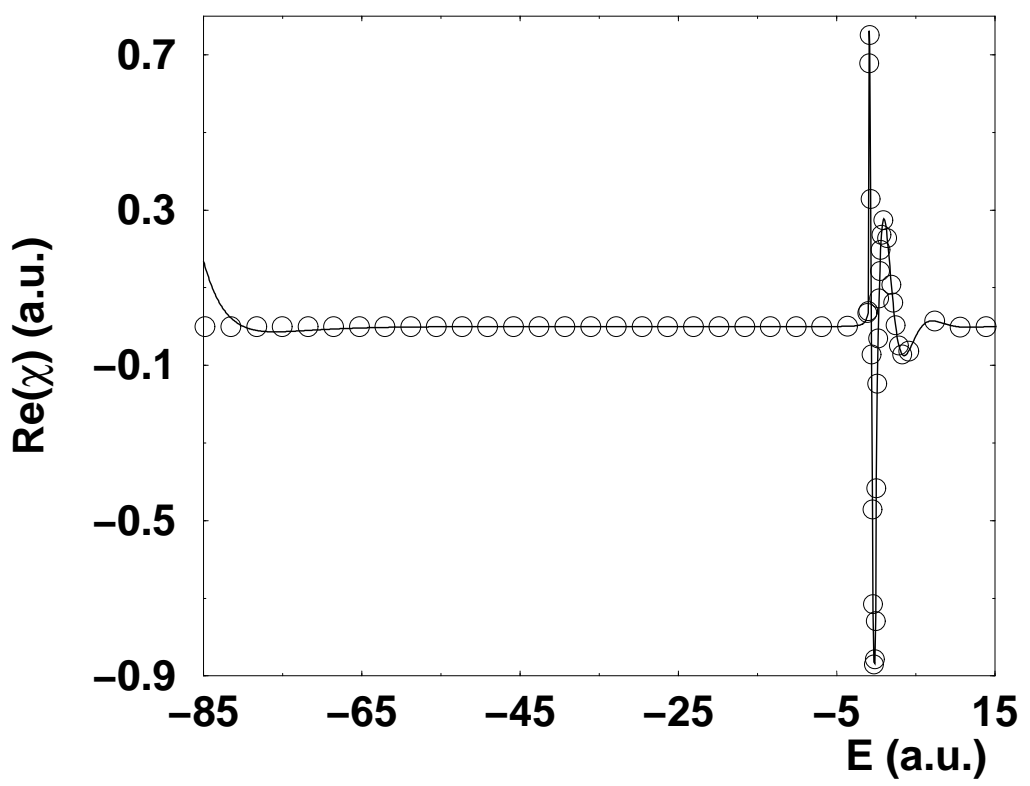

FIG. 6. Real parts of $\chi_{s}\left(E^{\prime}\right)$ (circles) and $\frac{m}{p} T^{l}(p) \tilde{\psi}_{\text {step }}^{\prime}(p)$ (solid line) for the step potential $\left(V_{0}=1\right)$. The initial state is a minimum uncertainty-product Gaussian with $\langle x\rangle=-3,\langle p\rangle=1$, and $\Delta_{x}=0.5$.

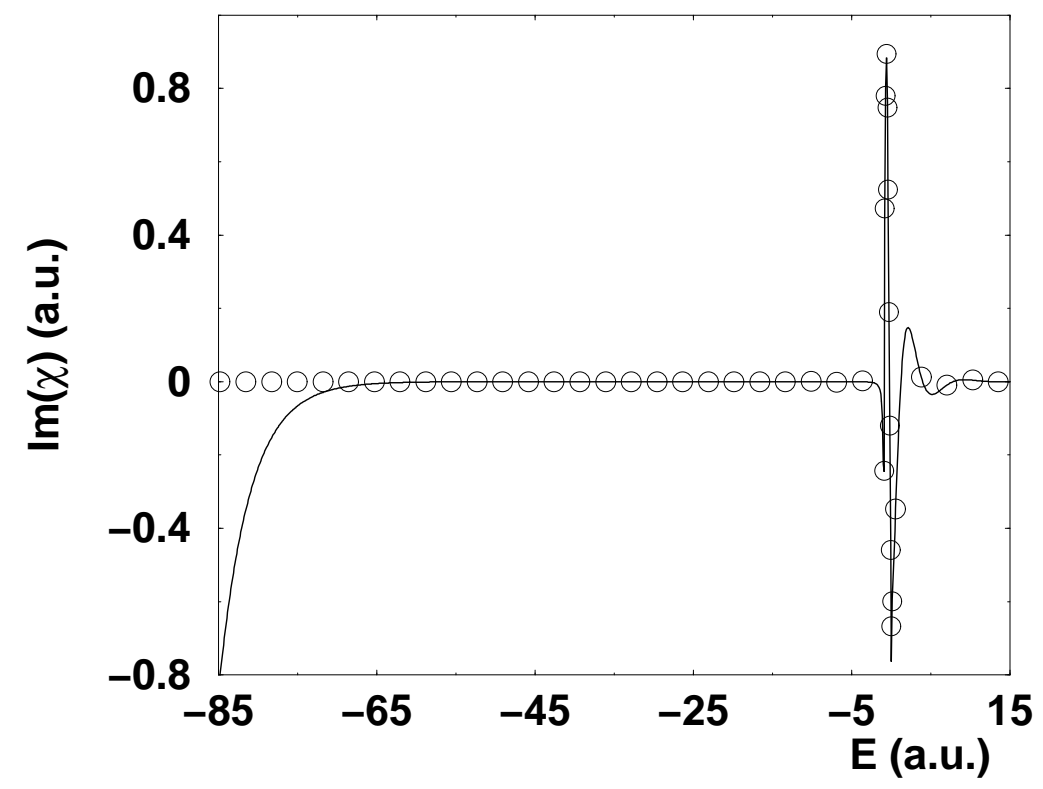

FIG. 7. Imaginary parts of $\chi_{s}\left(E^{\prime}\right)$ (circles) and $\frac{m}{p} T^{l}(p) \tilde{\psi}_{\text {step }}^{\prime}(p)$ (solid line) for the step potential $\left(V_{0}=1\right)$. Initial state as in Figure 6 .

\section{APPLICATION TO THE ARRIVAL TIME}

Allcock obtained Eq. (2) by assuming that Eq. (11) was satisfied, and that $V(x)=0$ for $x>0$, without any further specification of the potential after the initial state is released [1]. But each experimental setup will involve a particular potential profile, and we have seen that the interpretation of the source solutions in terms of an initial value problem depends on the particular potential that determines the dynamics. We shall in particular choose the simplest case of free motion to analyze Allcock's objection to the possibility of defining an ideal (apparatus independent) arrival-time concept in quantum mechanics based on Eq. (2). This section is an elaboration of the arguments given in [16].

Allcock's motivation for studying quantum mechanics with sources was the fact that the expression for the wave function, Eq. (2), involves an integral over energy from $-\infty$ to $\infty$. This suggested a possible way out to Pauli's 
argument against the existence of self-adjoint time operators conjugate to a semi-bounded Hamiltonian [17].

The core of Allcock's objection rests on the identification of the total arrival probability at a point $X>0, P(\infty)$, with the total amount of norm to the right of $X$ as $t \rightarrow \infty$,

$$
P(\infty) \equiv \lim _{t \rightarrow \infty} \int_{X}^{\infty} d x\left|\psi_{s}(x, t)\right|^{2}=\int_{0}^{\infty} d E\left|\left\langle E \mid \psi_{s}\right\rangle\right|^{2} .
$$

Here

$$
\left\langle E \mid \psi_{s}\right\rangle \equiv \chi_{s}(E)(2 E / m)^{1 / 4},
$$

where, as before, the branch cut for the fourth root is set at the negative imaginary axis. For free motion the second equality in Eq. (34) is to be expected since $\left\langle E \mid \psi_{s}\right\rangle(2 E / m)^{1 / 4}$, with $E>0$, is nothing but the amplitude for positive momentum $p$, so that we recover from Eq. (34) the known result

$$
\lim _{t \rightarrow \infty} \int_{X}^{\infty} d x\left|\psi_{s}(x, t)\right|^{2}=\int_{0}^{\infty} d p\left|\tilde{\psi}_{f}(p)\right|^{2} .
$$

Using two other independent routes Allcock arrives at integral expressions for a hypothetical total arrival time probability in contradiction with Eq. (34) because they have contributions from $E<0$; the simplest one is $\int_{-\infty}^{\infty} d E\left|\left\langle E \mid \psi_{s}\right\rangle\right|^{2}$. He therefore concludes "unequivocally that an ideal concept of arrival time cannot be established for the problem with sources $(-\infty<E<\infty)$ ". In the light of the identification between $\psi_{s}(x, t)$ and $\psi_{f}(x, t)$ in $D_{+}$made in section II, the flaw in Allcock's argument is that he is overlooking the contribution of negative momenta. According to classical prejudice one would not expect that negative momenta play any role for $t>0$ at $X>0$, if the initial state is confined within the left half-line. But quantum mechanics works otherwise, as Figures 1 and 2 clearly show. Since the negative energy part of $\psi_{s}(x, t)$ is equal to $\psi_{f,-}(x, t)$, we may equivalently think of the contribution of the negative momenta to a time-of-arrival probability in terms of evanescent waves. The negative energy components penetrate the positive coordinate region for some time before finally abandoning it. But Allcock's definition of $P(\infty)$ disregards this contribution entirely. Note that our criticism to the validity of $P(\infty)$ is rather independent on the theory chosen for defining an arrival time distribution. However, we cannot fail to point out that the equality between $\psi_{s}$ and $\psi_{f}$ in the space-time domain $D_{+}$allows immediately to associate Kijowski's distribution $\Pi_{K}$ [18, 16] to the source problem for free motion. This arrival time distribution has been extensively discussed elsewhere [19]21]. Similarly, for a general potential profile the generalization of $\Pi_{K}$ proposed in [20] is applicable.

\section{DISCUSSION}

The physical meaning of source boundary conditions used for the one dimensional, time dependent, single particle Schrödinger equation has been clarified by showing the equivalence of the corresponding solution $\psi_{s}$, see Eq. (2), with standard initial value problems for positive times and positions. We have first shown the connection between Eq. (2) and the ordinary initial-value-problem integral expression for a free motion wave packet, and then have extended this result for other potential profiles, in particular for the step barriers, which allow to inject waves dominated by evanescent energies into the right half-line.

Each of the potential profiles may correspond to a different experimental arrangement. Depending on the potential the origin of the evanescent waves in the source solution may be traced back to either negative momentum components (which are present in all cases, and become the only source of negative energies for free-motion), or "true" evanescent components, namely energy components which are already negative in the associated initial value problem, relative to the region of positive $x$. This occurs for example in the step potentials, where positive momenta with respect to the lower level become imaginary momenta in the upper level region. In addition to scattering contributions to the negative energies, we have also allowed for the possibility of bound state contributions, that may become important if the initial state overlaps with the normalized eigenstates of the Hamiltonian.

Apart from elucidating the physical content of previous works that have made use of source boundary conditions, our results have also served to analyze critically Allcock's negative statements about the possibility to define an ideal probability distribution of time of arrival for quantum waves with sources. It is argued that Allcock's postulated expression for a total arrival probability is flawed since it ignores the quantum contribution of negative momenta at positive positions and times. 


\section{ACKNOWLEDGMENTS}

We are grateful to José Pascual Palao for providing us with numerical tools. This work has been supported by Ministerio de Educación y Cultura (Grants PB97-1482 and AEN99-0315), The University of the Basque Country (Grant UPV 063.310-EB187/98), and the Basque Government (Grant PI-1999-28). A. D. Baute acknowledges an FPI fellowship by Ministerio de Educación y Cultura.

\section{APPENDIX A: SOLVABLE EXAMPLE}

We shall work out the time dependence of a state which is at $t=0$ the ground state of an infinite well of width $D$ between $a$ and $b=a+D$, and evolves freely from that time on. This was the only example of source mentioned by Allcock [1]:

$$
\psi_{i v}(x, t=0)=\left(\frac{2}{D}\right)^{1 / 2} \sin \left[(x-a) k_{w}\right] \mathcal{H}(a, b)
$$

where $k_{w}=\pi / D$, and

$$
\mathcal{H}(a, b)=\left\{\begin{array}{ll}
1 & a<x<b \\
0 & \text { otherwise }
\end{array} .\right.
$$

Eq. (A1) may also be written as a combination of four plane waves truncated at $a$ and $b$, two of them with momentum $p_{w}=k_{w} \hbar$, and the other two with momentum $-p_{w}$. The time evolution of a truncated plane wave was first solved by Moshinsky [22]. For an alternative derivation see [10]. The resulting wave is given by

$$
\psi_{i v}(x, t)=\frac{1}{4 i}\left(\frac{2}{D}\right)^{1 / 2}\left\{e^{i k_{w} D} e^{i m(x-b)^{2} / 2 t \hbar}\left[w\left(-u_{b+}\right)-w\left(-u_{b-}\right)\right]+e^{i m(x-a)^{2} / 2 t \hbar}\left[w\left(-u_{a-}\right)-w\left(-u_{a+}\right)\right]\right\},
$$

where

$$
\begin{aligned}
u_{b \pm} & =\frac{ \pm p_{w}-m(x-b) / t}{f}, \\
u_{a \pm} & =\frac{ \pm p_{w}-m(x-a) / t}{f} \\
f & =(1-i)\left(\frac{m \hbar}{t}\right)^{1 / 2}, \\
w(u) & =e^{-u^{2}} \operatorname{erfc}(-i u) .
\end{aligned}
$$

For properties of the $w$-function see [23]. The momentum representation of Eq. (A1) is also of interest to illustrate the properties mentioned in section II (analyticity and decay to zero in the upper half plane when the state is confined between two points in coordinate space),

$$
\tilde{\psi}_{i v}(p)=-\frac{(2 h)^{1 / 2}}{4 \pi D^{1 / 2}} \sum_{\alpha= \pm} \alpha \frac{e^{-i\left(p+\alpha p_{w}\right) b / \hbar}-e^{-i\left(p+\alpha p_{w}\right) a / \hbar}}{p+\alpha p_{w}} e^{i \alpha p_{w} a / \hbar} .
$$

[1] G. R. Allcock, Ann. Phys. (N.Y.) 53, 253 (1969).

[2] K. Stevens, Eur. J. Phys. 1, 98 (1980).

[3] K. Stevens, J. Phys. C: Solid State Phys. 16, 3649 (1983).

[4] P. Moretti, Phys. Scripta 45, 18 (1992).

[5] A. Ranfagni, D. Mugnai, and A. Agresti, Phys. Lett. A158, 161 (1991). 
[6] M. Büttiker and H. Thomas, Ann. Phys. (Leipzig) 7, 602 (1998).

[7] M. Büttiker and H. Thomas, Superlattices and Microstructures 23, 781 (1998).

[8] J. G. Muga and M. Büttiker, (2000), quant-ph/0001039, Phys. Rev. A to appear.

[9] M. Mangin-Brinet, J. Carbonell, and C. Gignoux, Phys. Rev. A57, 3245 (1998), quant-ph/9804030.

[10] S. Brouard and J. G. Muga, Phys. Rev. A54, 3055 (1996).

[11] A. D. Baute, I. L. Egusquiza, and J. G. Muga, submitted.

[12] The cut-off condition is not strictly necessary for the left edge of the potential. The analyticity of $T(p)$ (except possibly at simple bound state poles on the imaginary axis) and the limit $T(p) \rightarrow 1$ as $|p| \rightarrow 1$ in the upper half plane hold for potentials that satisfy $\int_{-\infty}^{\infty} d x\left(1+x^{2}\right)|V(x)|<\infty$ 14.

[13] L. Faddeev, Trudy Mat. Inst. Steklov 73, 314 (1964), Am. Math. Soc. Transl. 2 (1964) 139.

[14] R. G. Newton, J. Math. Phys. 21, 493 (1980).

[15] M. S. Marinov and B. Segev, J. Phys. A29, 2839 (1996), quant-ph/9602015.

[16] J. G. Muga and C. R. Leavens, Arrival time in quantum mechanics, Phys. Rep. to appear.

[17] In fact Pauli's argument is not as fierce as it seems. Apart from the technical flaws emphasized by E. Galapon (quantph 9908033, 0001062), time observables may be associated with Positive Operator Valued Measures (POVMs), without requiring that the first moment operator of the POVM be self-adjoint [16], see also M. D. Srinivas and R. Vijayalakshmi, Pramana 16, 173 (1981); P. Busch, M. Grabowski, and P. J. Lahti, Phys. Lett. A 191, 357 (1994).

[18] J. Kijowski, Rept. Math. Phys. 6, 361 (1974).

[19] J. G. Muga, C. R. Leavens, and J. P. Palao, Phys. Rev. A58, 1 (1998), quant-ph/9807066.

[20] A. D. Baute, R. Sala-Mayato, J. P. Palao, J. G. Muga, and I. L. Egusquiza, Phys. Rev. A61, 022118 (2000), quant$\mathrm{ph} / 9904055$.

[21] I. L. Egusquiza and J. G. Muga, Phys. Rev A61, 012104 (2000), quant-ph/9905023.

[22] M. Moshinsky, Phys. Rev. 88, 625 (1952).

[23] M. Abramowitz and I. Stegun, Handbook of Mathematical Functions (Dover, New York, 1972). 